\title{
SliceNet: End-to-End Cognitive Network Slicing and Slice Management Framework in Virtualised Multi-Domain, Multi-Tenant 5G Networks
}

\author{
Qi Wang ${ }^{1}$, Jose Alcaraz-Calero ${ }^{1}$, Maria Barros Weiss ${ }^{2}$, Anastasius Gavras ${ }^{2}$, Pedro Miguel Neves ${ }^{3}$, Rui Cale ${ }^{3}$, Giacomo \\ Bernini $^{4}$, Gino Carrozzo ${ }^{4}$, Nicola Ciulli ${ }^{4}$, Giuseppe Celozzi ${ }^{5}$, Angelo Ciriaco ${ }^{5}$, Anna Levin ${ }^{6}$, Dean Lorenz ${ }^{6}$, Katherine \\ Barabash $^{6}$, Navid Nikaein ${ }^{7}$, Salvatore Spadaro ${ }^{8}$, Donal Morris ${ }^{9}$, Ioannis Chochliouros ${ }^{10}$, Yiorgos Agapiou ${ }^{10}$, Cristian \\ Patachia $^{11}$, Marius Iordache ${ }^{11}$, Elena Oproiu ${ }^{11}$, Cipriano Lomba ${ }^{12}$, Ana Cristina Aleixo ${ }^{12}$, Alberto Rodrigues ${ }^{12}$, Ger \\ Hallissey ${ }^{13}$, Zdravko Bozakov ${ }^{13}$, Konstantinos Koutsopoulos ${ }^{14}$, Paul Walsh ${ }^{15}$ \\ ${ }^{1}$ University of the West of Scotland, UK; ${ }^{2}$ Eurescom GmbH, Germany; ${ }^{3}$ Altice Labs, Portugal; ${ }^{4}$ Nextworks, Italy; ${ }^{5}$ Ericsson \\ Telecomunicazioni, Italy; ${ }^{6}$ IBM, Israel; ${ }^{7}$ EURECOM, France; ${ }^{8}$ Universitat Politecnica de Catalunya, Spain; ${ }^{9}$ RedZinc Services \\ Ltd., Ireland; ${ }^{10} \mathrm{OTE}$, Greece; ${ }^{11}$ Orange Romania; ${ }^{12}$ Efacec Energia, Portugal; ${ }^{13}$ DellEMC, Ireland; ${ }^{14}$ Creative Systems \\ Engineering, Greece; ${ }^{15} \mathrm{CIT}$ INFINITE, Ireland
}

\begin{abstract}
Network slicing has emerged as a major new networking paradigm for meeting the diverse requirements of various vertical businesses in virtualised and softwarised $5 \mathrm{G}$ networks. SliceNet is a project of the EU 5 G Infrastructure Public Private Partnership (5G PPP) and focuses on network slicing as a cornerstone technology in 5G networks, and addresses the associated challenges in managing, controlling and orchestrating the new services for users especially vertical sectors, thereby maximising the potential of $5 \mathrm{G}$ infrastructures and their services by leveraging advanced software networking and cognitive network management. This paper presents the vision of the SliceNet project, highlighting the gaps in existing work and challenges, the proposed overall architecture, proposed technical approaches, and use cases.
\end{abstract}

Keywords-5G; network slicing; slice management; cognitive network management; verticals

\section{INTRODUCTION}

It has now been widely recognised that the softwarenetworking based architecture approach with NFV (Network Function Virtualisation) and SDN (Software-Defined Networking) as main enablers is essential in achieving the $5 \mathrm{G}$ vision of being "programmable, software driven and holistically managed to enable a diverse range of services in a profitable way", as summarized by the Chairman of the 5GPPP Association [1]. In particular, network slicing has emerged as a most promising game changer in the remarkable paradigm shift from $4 \mathrm{G}$ to NFV/SDN-enabled $5 \mathrm{G}$ era. According to the visions by various leading $5 \mathrm{G}$ Standards Developing Organisations (SDOs) and other stakeholders, network slicing has been identified as a crucial enabler for provisioning flexible, cost-efficient and tailored services in softwarenetworking based $5 \mathrm{G}$ networks.

The expected benefits of introducing Network Slicing in $5 \mathrm{G}$ are multi-folded. Firstly, network slices would maximize the sharing of network resources within and across domains, thereby substantially reducing the capital expenditure for network operators. Secondly, network slicing allows a high- degree of flexibility for creating dedicated logical networks with customer-specific functions and thus can meet diverging requirements from diverse vertical businesses. Thirdly, network slices can substantially upgrade operational capabilities as with intelligent slicing and slice lifecycle management it is possible to offer configurable guarantees in Quality of Service (QoS) or Quality of Experience (QoE). These configurable guarantees in service quality will open a significant number of new markets and enable a wide range of demanding, divergent and innovative use cases. For instance, demanding eHealth applications would become a reality where an Ultra-High-Definition video stream can be transmitted with the guaranteed ultra-low latency and mobile broad bandwidth. Therefore, if the full potential is achieved, network slicing could be one of the most important innovations in the $5 \mathrm{G}$ era due to its impact at worldwide level.

The reminder of the paper is structured as follows. Section II reviews the state of the art and highlights the gaps on 5G network slicing. Section III presents the SliceNet architecture in addressing the technical gaps identified. Section IV describes the project's technical approaches to achieve advances beyond the state of the art in SliceNet. Section V outlines the primary use cases in the project. The paper is concluded in Section VI, which also provides a perspective of ongoing and future work of the project.

\section{EXISTING GAPS AND TECHNICAL CHALLENGES}

In light of the rapidly growing recognition of the significance of network slicing, major European and worldwide telecommunication operators and network equipment vendors have declared the importance of achieving network slicing as a fundamental architectural requirement and critical enabler of 5G networks (e.g., Orange [2]). Moreover, the last couple of years have witnessed an increasing number of headlines, highlighting pioneering prototypes or early trials of network slicing by leading operators or vendors. Examples include "Ericsson and SK Telecom demonstrate 5G network slicing technology" [3], "DOCOMO and Ericsson perform successful 
Proof of Concept of dynamic 5G network slicing" [4], and "Huawei and Deutsche Telekom Demonstrate 5G End-to-End (E2E) Network Slicing Technology" [5], among a few other trials.

Despite these encouraging efforts, there are a number of gaps that need to be filled to fully realise the envisioned benefits of $5 \mathrm{G}$ slices. Firstly, E2E slicing has been specified by ITU [6] as a high-priority technical gap to tackle for E2E application quality; however, the reported prototypes are not truly E2E network slicing across different administrative domains and far from realising large-scale operation. For instance, the operation of the joint Huawei and Deutsche Telekom E2E slicing prototype was limited to the Deutsche Telekom lab, although the latest advance in an inter-continental trial [7] has showed more promise. Moreover, most of these prototypes only work when the users subscribe to the same network operator and in fact in most cases only when the users are connected to the same model of antennas from the same vendor. Currently, there is a clear gap on the extension of the network slicing from one single administrative domain to a scenario where multiple providers are cooperating to achieve truly E2E network slicing across multiple administrative domains. Due to the technical challenges, this multi-domain slicing has yet to be fully achieved.

Secondly, existing projects focus on the control plane, i.e., slicing prototyping. No existing projects have positioned to focus on the management plane of slices. The 5GEx project [8] is the only one out of $195 \mathrm{G}$ PPP Phase I projects that explicitly covers a multi-domain aspect of 5G systems. 5GEx is a first step towards the achievement of the ambitious E2E network slicing capabilities of the $5 \mathrm{G}$ systems, in terms of providing the first prototypes of the drivers for the control plane to analyse the technical viability of a network slicing capability of the data plane [9].

Thirdly, recent vertical markets' analyses and related studies [10] have shown the importance of forming a close partnership between $5 \mathrm{G}$ industry and vertical business sectors in achieving the fully connected society vision in $5 \mathrm{G}$. However, existing $5 \mathrm{G}$ projects have rarely given emphasis on facilitating vertical businesses to employ 5G (let alone codesign 5G) in a flexible and efficient fashion. For instance, 5G PPP Phase I projects mainly take a technology-driven approach and focus on enabling 5G technologies, whilst 5G PPP Phase II projects are expected to collaborate much closer with vertical businesses to promote $5 \mathrm{G}$. It is thus essential to offer a costeffective migration pathway for verticals to adopt $5 \mathrm{G}$ slicebased/enabled services.

Finally, the QoE of users of $5 \mathrm{G}$ slices has yet to be addressed. Upon achieving slicing, the next critical step and major challenge is to maintain or even improve the applicationlevel quality for the use cases of the vertical businesses, which can be achieved through dynamically optimising the perceived quality of the running slices. QoE should not be achieved via over-provisioning of QoS, which is expensive and not scalable.

Motivated by the above context, SliceNet targets to drive $5 \mathrm{G}$ network slicing up to the next level, by pushing the

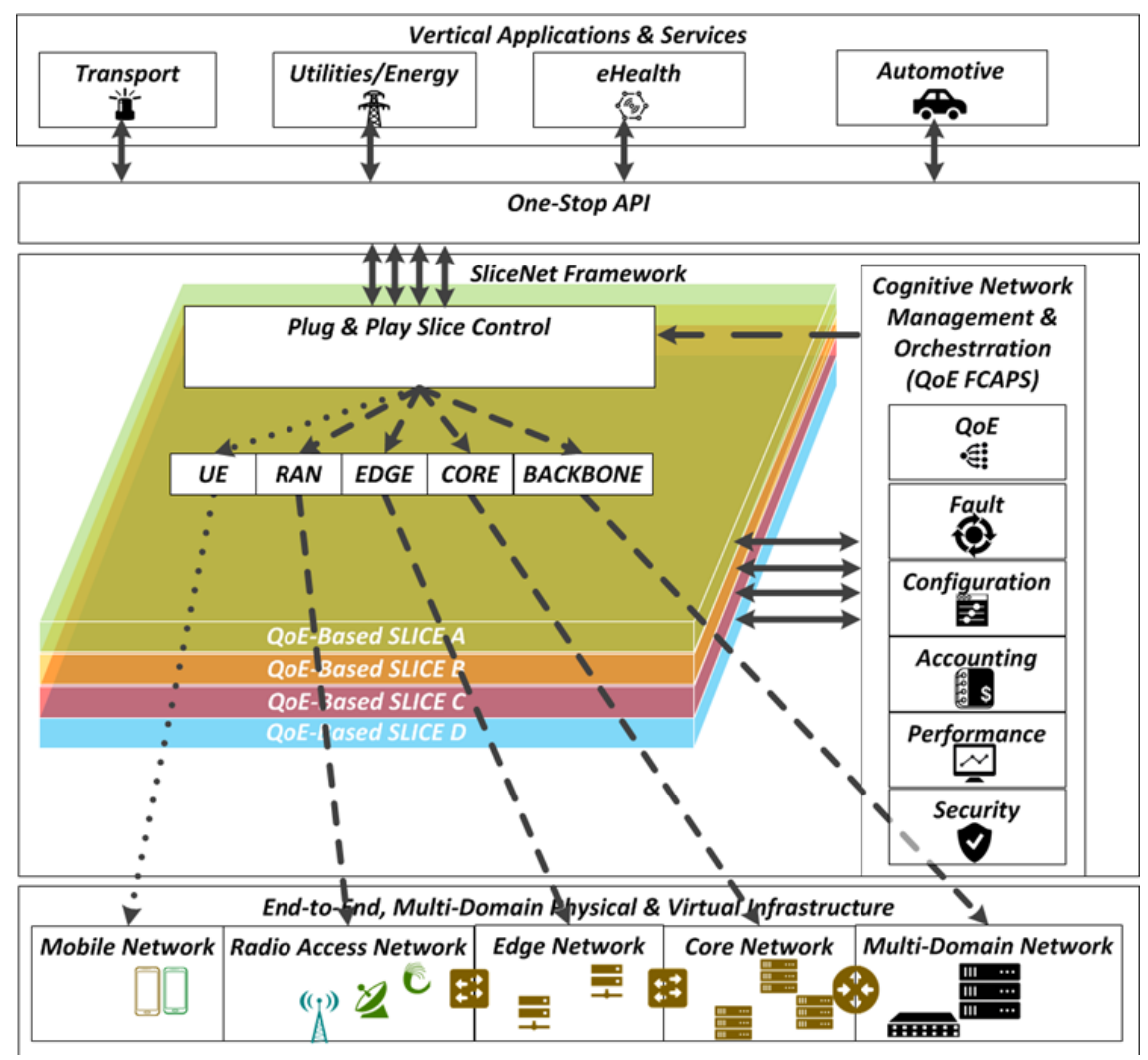

Figure 1 SliceNet Vision 
boundaries significantly in fulfilling the challenging requirements from the management and control planes of network slicing across multiple administrative domains, facilitating early and smooth adoption of $5 \mathrm{G}$ slices for verticals to achieve their demanding use cases, and managing the QoE for slice services. To realise this highly ambitious vision, a novel and powerful integrated network management, control and orchestration framework is entailed for realising $5 \mathrm{G}$ network slicing and slice-based enabled services.

\section{Proposed SLICENET ARChITECTURE}

\section{A. Project Vision}

Figure 1 shows the overall vision for SliceNet where the aim and objectives are represented. SliceNet aims to design, prototype and demonstrate an innovative, verticals-oriented, QoE-driven 5G network slicing framework focusing on cognitive network management and control for end-to-end slicing operation and slice-based/enabled services across multiple operator domains in SDN/NFV-enabled 5G networks. SliceNet will tackle a number of highly challenging outstanding issues in $5 \mathrm{G}$ network slicing in terms of finegrained and integrated management, QoE modelling \& management, customisable control, cross-plane coordination and orchestration, slicing scalability, security, resource efficiency and interoperability across multiple domains.

\section{B. System Architecture}

The overall architecture of SliceNet is composed mainly by two architectural domains. One is the advanced managed domain (infrastructure, services and control) imposed by the challenging requirements of $5 \mathrm{G}$ systems where network softwarisation and slicing, SDN, NFV, and other architectural designs and principles for $5 \mathrm{G}$ performances will be fully integrated and thus establish an integrated, softwarised, slicingready infrastructure for $5 \mathrm{G}$ services in SliceNet. The other is the innovative management domain (management and orchestration) that is able to cognitively manage this novel infrastructure and its new services by addressing a set of technological challenges such as multi-tenancy, multi-operator, multi-domain, programmable data plane, pluggable control plane and cross-layer orchestration. Figure 2 shows an overview of the SliceNet architecture where this division between the managed infrastructure and the management architecture is depicted. SliceNet will follow a layered architectural approach to allow the creation of a modular, extensible and scalable framework. The SliceNet managed domain consists of five layers, as shown in Figure 2.

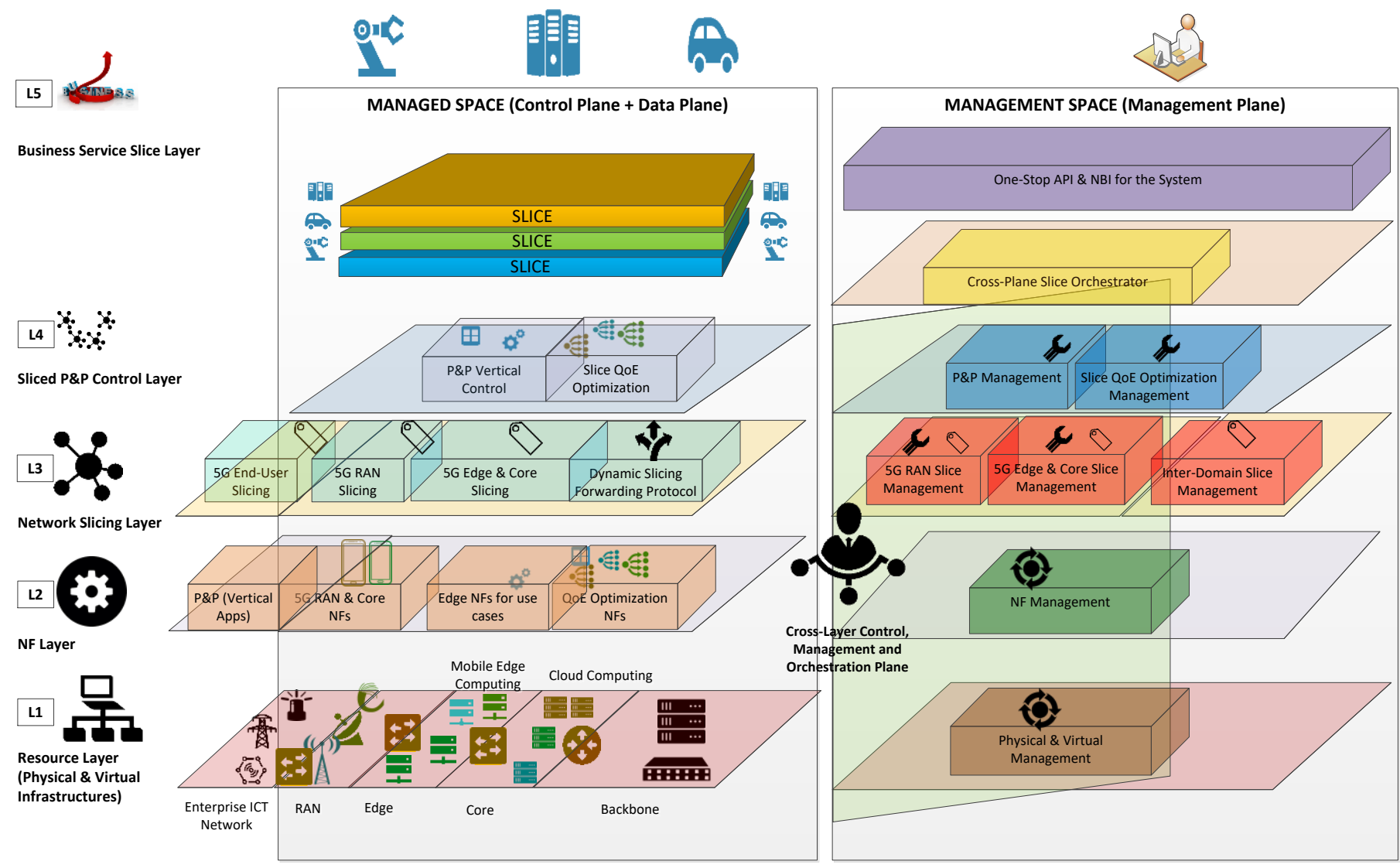

Figure 2 SliceNet Overall Architecture 
From a bottom-up perspective, the first layer (L1 in Figure 2 ) is the Infrastructure Layer, representing the $5 \mathrm{G}$ physical and vertical infrastructure deployments. Cloud computing technologies are utilised and the Mobile Edge Computing paradigm is integrated to push virtualised resources to the edge of the network. The Network Function (NF) Layer (L2) comprises all the services (mainly NFV services in SliceNet) running on top of the underlying infrastructure. The Network Slicing Layer (L3) is decoupled from the NF Layer following the SDN paradigm to gain a holistic, logically centralised control of the geographically distributed services. The Sliced Plug \& Play (P\&P) Control Layer (L4) is a new layer introduced by SliceNet as a novel extension of the multi-tenant concept now embracing the SDN world. This layer will provide a novel P\&P functionality of the control plane so that it is customised and isolated for a particular vertical customer differentiating itself from the common control plane for all the users of the infrastructure. This layer enables hosting P\&P control applications provided by the vertical business, for example a QoE Optimization application to fulfil the requirements of a given vertical business. Finally, the Business Service Slice Layer (L5) provides the highly customisable Slice-as-a-Service to a range of vertical businesses with diverging use case requirements.

\section{TeChNiCAl ApPRoACHES AND AdVANCES Beyond THE STATE OF THE ART}

The proposed SliceNet project will significantly advance the state of the art (SOTA) in cognitive, autonomic, QoEdriven network management, control and orchestration of softwarised $5 \mathrm{G}$ systems. The main innovation of SliceNet is the highly novel, cognition-based, fully integrated SliceNet architecture and framework that meets the challenging nextgeneration network management visions and requirements from various $5 \mathrm{G}$ stakeholders.

For 5G network operators, SliceNet advances the SOTA in enabling a truly end-to-end multi-domain network management paradigm with integrated Fault, Configuration, Accounting, Performance and Security (FCAPS) management. For 5G service providers and users, SliceNet advances the SOTA in achieving significantly higher service quality towards warranted perceived quality through the intelligent QoE-driven design. For 5G vertical businesses, SliceNet advances the SOTA in facilitating the verticals to employ $5 \mathrm{G}$ services in a much more rapid and efficient manner through a 'one-stopshop' approach.

Above all, SliceNet will deliver a 5G network slicing innovation ecosystem, centred on promoting and advancing network slicing-based softwarisation of $5 \mathrm{G}$ systems in architectural enhancements and infrastructure integration, as well as novel enablers for the data plane, control plane, management plane and cross-plane orchestration. The SliceNet framework will be completed with standards-compliant interfaces and new, open and extensive Application Programming Interfaces (APIs) to fulfil the overall aim proposed for SliceNet, enabling early adoption and facilitating a wider deployment of network slicing and slice-based or enabled services, applications and thus opening new market opportunities.
Aligned with the highly ambitious objectives of 5G PPP, SliceNet is featured with a set of novel concepts and technical approaches, leading to highly demanded or desirable new services and potential new business models and products, highlighted below wherever applicable, in line with the common and private exploitation plans from SliceNet partners. In SliceNet, the following specific advances beyond the SOTA and the main technical approaches that enable these advances are expected:

- In the data plane, SliceNet explores the programmability of the data paths across the various network segments in the E2E network slice, and investigates virtualised slicing-friendly infrastructure.

- In the control plane, SliceNet targets to achieve truly multiple domain cooperation from multiple network operators, vendors and service providers, leading to new large-scale 5G services and new federation business models. The experiences learnt from Phase I project 5GEx will be exploited to advance this ambitious multidomain slicing vision.

- In the management plane, SliceNet plans to deliver a new set of integrated FCAPS slice management functionalities, leading to new FCAPS products for $5 \mathrm{G}$ slices management. On top of this, SliceNet proposes a novel cognition-based QoE management approach, and will deliver new QoE sensors and actuators, leading to potentially new products. The cognitive network management approach will leverage related results from Phase I projects especially SELFNET and CogNet.

- In the orchestration plane, SliceNet takes a novel crossplane coordination approach, and will deliver a novel cross-plane orchestrator that also preserves the boundaries among the planes, leading to potentially new orchestrator product to be integrated by the network operators, in line with their exploitation plans.

- SliceNet takes a novel 'Verticals in the loop' design approach for the entire SliceNet framework (not just the use cases but also Plug \& Play control, FCAPS etc.), and will deliver the innovative 'one-stop-shop' 5G slicebased/enabled service solution for verticals to efficiently upgrade existing or creating new use cases the SliceNet northbound interface. The customisation of slices featured with the novel Plug \& Play control plane is a new service, leading to new business models between the verticals and the slice service provider.

- SliceNet will prototype three 5G-enabled use cases demonstrations, contributing to EU-wide ambitious Phase II trials objective. The three carefully selected use cases in SliceNet are 5G Smart Grid Self-Healing, 5G eHealth Connected Ambulance and 5G Smart City Smart Lighting, which represent Energy/Utilities, Health and Smart City respectively, among the top vertical industries identified by EC as the most promising/influential $5 \mathrm{G}$ users. These use cases are highly ambitious in QoS/QoE requirements (e.g., ultra-high reliability, quality of information and experience, and ultra-low latency etc.), challenging 5G slicing capabilities to its limit. 


\section{USE CASES}

Three primary use cases are planned in the project to validate the effectiveness and demonstrate the benefits of the SliceNet technical approaches in an integrated environment. A brief description of these three use cases are presented below:

5G Smart Grid Self-Healing Use Case: The automation in energy distribution with self-healing solutions is a critical challenge to consider in the transformational evolution towards Smart/Smarter Grid. Self-healing will enable system operators to benefit from a significant reduction in the outage duration, in the number of affected customers as well as in the number of switching manoeuvres required during network reconfiguration procedure involved in Fault Detection Isolation and Service Restoration. Timely, reliable and secure communications are crucial in this use case.

5G eHealth Smart/Connected Ambulance Use Case: The Connected Ambulance will act as a connection hub for the emergency medical equipment and wearables, enabling realtime streaming to the awaiting emergency department team at the destination hospital. This use case will advance the emergency ambulance services by developing new collaborative models with the healthcare stakeholders to help create improved experiences and outcomes for patients in their care. Both single and multi-patient scenarios will be explored to maximise the impact of $5 \mathrm{G}$ technology on patient care.

Smart City SmaLI-5G Use Case: Intelligent Public Lighting system in a smart city will enable remote control in real time and in a secure way every single lighting pole from the target network, in order to adjust the lighting intensity and efficiently manage energy consumption (reduction of energy costs for up to $80 \%$ ). Moreover, the system, based on a high-level architecture constructed on three levels: data collection and transport layer, open IoT middleware layer and application layer, will allow real-time and history-based energy consumption measuring. Furthermore, the entity responsible with the streets lighting infrastructure operation and maintenance will be able to proactively spot the malfunctions, energy loss or energy theft tentative on the public lighting network.

\section{CONCLUSION}

Network slicing is a crucial enabler for $5 \mathrm{G}$ networks through creating multiple independent logical networks over the same physical infrastructure. The SliceNet project presented in this paper targets to remove the limitations of current network infrastructures by achieving fully softwarisation-friendly 5G infrastructures, and radically address the associated challenges in managing, controlling and orchestrating the new services running in such infrastructures for users (especially vertical sectors), thereby maximising the potential of $5 \mathrm{G}$ infrastructures and their services based on advanced software networking and cognitive network management.

Ongoing work has been focusing on defining the detailed functional architecture for the overall SliceNet framework and specifying the components and their interactions in the involved planes in this architecture. Future activities will prototype the key enablers in the system for achieving network slicing and its control and management.

\section{ACKNOWLEDGMENT}

This work has been funded in part through the European Union's H2020 program, under grant agreement No 761913: project SliceNet. The authors would like to thank all SliceNet partners for their support in this work.

\section{REFERENCES}

[1] (2018) [Online] Available at http://telecoms.com/429031/5g-consortium-commencesnovel-network-architecture-initiative/

[2] Orange, “Orange 5G Vision”, Sept. 2016, [Online] available at

http://www.orange.com/fr/content/download/38314/1162 326/version/2/file/Orange\%205G\%20vision\%20(en\%20a nglais, \%20septembre\%202016).pdf

[3] Ericsson, "Ericsson and SK Telecom demonstrate 5G network slicing technology", Oct. 2015, [Online] available at https://www.ericsson.com/news/151029ericsson-and-sk-telecom 244069644 c.

[4] Ericsson, "DOCOMO and Ericsson perform successful Proof of Concept of dynamic 5G network slicing", Jun. 2016, available at

https://www.ericsson.com/news/160613-DOCOMOproof-of-concept-of-dynamic-5G-networkslicing 244039855_c.

[5] Huawei, "Huawei and Deutsche Telekom Demonstrate 5G E2E Network Slicing Technology", Sept. 2016, [Online] available at http://www.huawei.com/en/news/2016/2/Demonstrate5G-E2E-Network-Slicing-Technology.

[6] ITU-T Focus Group IMT-2020, "Report on Standards Gap Analysis", Dec. 2015, [Online] available at http://www.itu.int/en/ITU-T/focusgroups/imt2020/Pages/default.aspx.

[7] "Deutsche Telekom, SK Telecom use network slicing to trial 5G across continents", Mar. 2018, [Online] available at https://mobileeurope.co.uk/press-wire/deutschetelekom-sk-telecom-use-network-slicing-to-trial-5 gacross-continents.

[8] (2017) 5GEx project, [Online] available at http://www.5gex.eu/.

[9] (2016) 5GEx white paper, [Online] available at http://www.5gex.eu/wp/wpcontent/uploads/2016/03/5GEx-White-Paper-v1.pdf.

[10] 5G PPP, "5G empowering vertical industries", Feb. 2016, [Online] available at https://5g-ppp.eu/wpcontent/uploads/2016/02/BROCHURE_5PPP_BAT2_PL. pdf.

[11] SELFNET, Mar. 2018, [Online] available at https://selfnet- $5 \mathrm{~g} . \mathrm{eu} /$.

[12] CogNet, Mar. 2018, [Online] available at http://www.cognet.5g-ppp.eu/. 